\title{
Pitfalls in the Management of Atlanto-Occipital Dislocation
}

\author{
Masahiro Aoyama, Muneyoshi Yasuda, Masahioro Joko, \\ Mikinobu Takeuchi, Aichi Niwa, Masakazu Takayasu \\ Department of Neurosurgery, Aichi Medical University, Nagakute, Japan
}

\begin{abstract}
Atlanto-occipital dislocation (AOD) is rarely seen in clinic because it is characteristically immediately fatal. With recent progress in the pre-hospital care, an increasing number of AOD survivors have been reported. However, because the pathophysiology of AOD is not clearly understood yet, the appropriate strategy for the initial management remains still unclear. We report a case of successful AOD treatment and describe important points in the management of this condition. It is important to note that abducens nerve palsy is a warning sign of AOD and that AOD can result in a life-threatening distortion of the arteries and the brain stem. We recommend the application of a halo vest to protect the patient's neural and vascular competence as the immediate initial step in the treatment of AOD. Horn's grading system is useful in assessing indications for surgery. Finally, when performing posterior fixation, C2 should be included because of the anatomy of the ligamentous architecture.
\end{abstract}

Keywords: Atlanto-occipital joint; External fixators; Internal fixators

\section{Introduction}

The atlanto-occipital junction serves as a supportive base for the skull and contains vital structures such as the vertebral arteries and the spinal cord. Although this junction bears the heavy load of the head, it is also a pivot point for head motion. Therefore, the joint is flexible in every direction and has loose its bone connections. Many ligaments surround it, maintaining its stability [1]. Because of its anatomy, the atlanto-occipital junction is vulnerable to a dislocation in a high-impact trauma. An atlanto-occipital dislocation (AOD) has long been a rare clinical entity because of its associated rapid mortality [2,3]. However, the number of AOD survivors is increasing with recent developments in the emergency medical care [1,2,4-6]. Herein, we report the successful treatment of a patient with $\mathrm{AOD}$ and discuss the appropriate management strategy.

\section{Case Report}

A 33-year-old male engineer fell $10 \mathrm{~m}$ while working on the roof of a factory and was immediately transported on a spine board by a helicopter emergency medical service with trained medical staff to our hospital. Upon arrival, his vital signs were stable. His Glasgow coma scale (GCS) score was E2V(T)M6 with normal pupillary findings. Although he did not respond to verbal commands, he retained rectal tone.

The radiological examination showed multiple injuries, including contusions of the liver and lung and fractures of the scapulae and pelvis. A remarkable retropharyngeal swelling was evident on cervical X-ray analyses (Fig. 1A).

Received Mar 27, 2014; Revised Jun 12, 2014; Accepted Jun 12, 2014

Corresponding author: Masahiro Aoyama

Department of Neurosurgery, Aichi Medical University, 1-1 Yazakokrimata, Nagakute 480-1195, Japan

Tel: +81 561623311, Fax: +81 561632879, E-mail: maoyama@aichi-med-u.ac.jp 
The computed tomography (CT) showed a translocation of both occipital condyles anterior to the lateral masses of the atlas (Fig. 1B). Numeric measurements were consistent with the published criteria for AOD [3]. In addition, the magnetic resonance imaging (MRI) showed a compression of the spinal cord by a hematoma (Fig. 1C, D). The digital subtraction angiography (DSA) showed the internal carotid and vertebral arteries to be stretched bilaterally because of a distraction between the atlas and occiput (Fig. 1E).

On the day of admission, an interventional embolization was performed to achieve hemostasis in the contused organs (Fig. 2A, D). The patient was fitted with a hard collar. On the second day, a halo orthosis was fitted to augment cervical immobilization. The patient gradually regained consciousness (GCS, E3V[T]M6) but exhibited severe motor weakness with a manual muscle test (MMT) score of $0 / 5$ for the whole body except the bilateral toes, which retained their flexion and extension and had a MMT score of 4/5. On the third day (Fig. 2B, E), the right toe's MMT score dropped to 1/5 and C3-4 laminectomy and hematoma removal was performed. The right $\mathrm{C} 4$ and 5 nerve roots were found to be severely avulsed. As the dura mater was partially destroyed, a piece of fascia was used to cover it. After surgery, the patient became drowsy and exhibited signs of progressive abducens nerve palsy. The monitoring of the cerebral regional saturation of oxygen $\left(\mathrm{rSO}_{2}\right)$ suggested a decreased blood flow to the brain; the $\mathrm{rSO}_{2}$ showed $78 \%$ on the first day and $67 \%$ on the third day. Routine follow-up CT scans showed an exacerbation of the AOD. Therefore, based on the CT scans, we adjusted the halo vest twice with vertical compressive
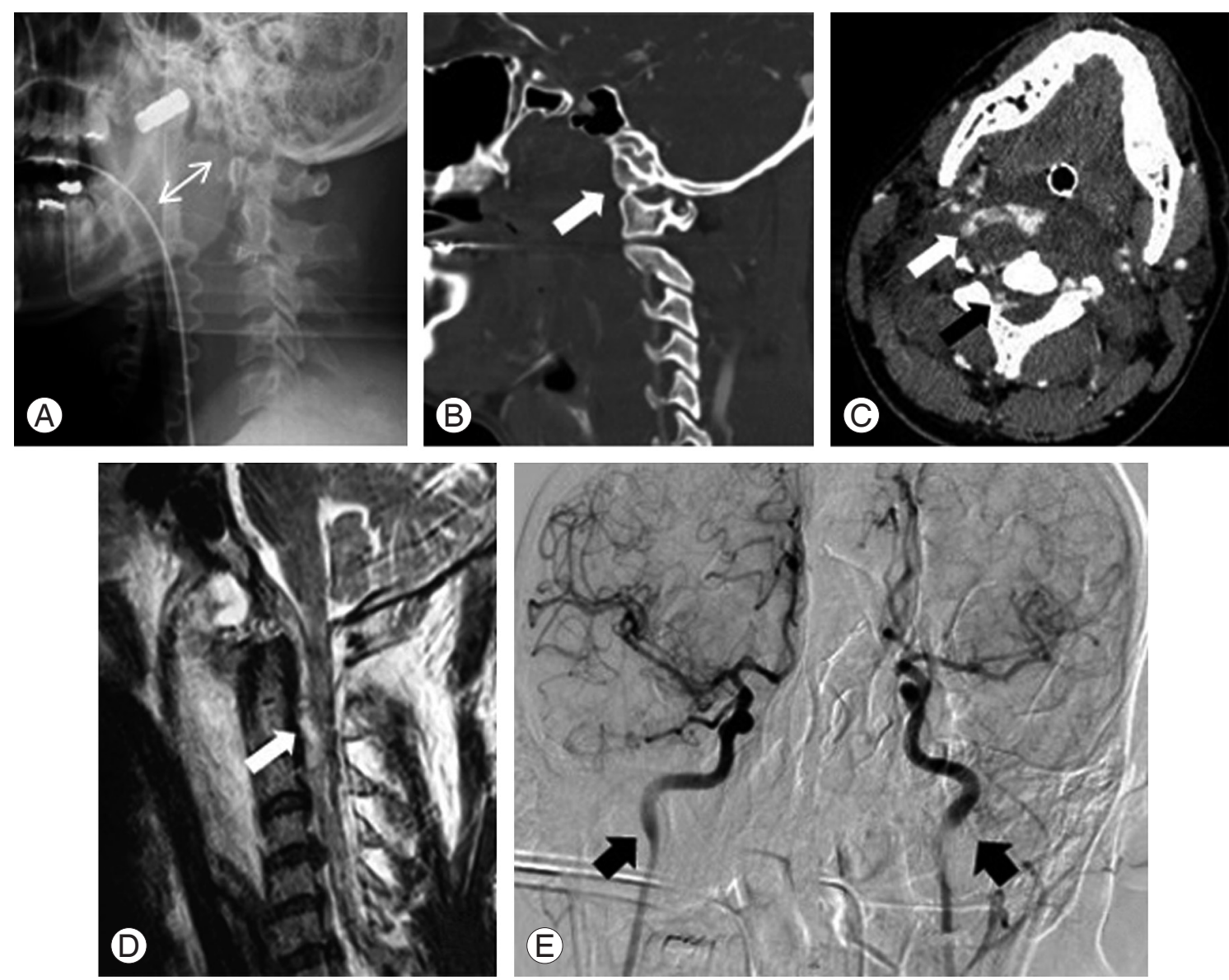

Fig. 1. Radiological findings on the day of transfer to our hospital. (A) Lateral X-ray results showing a marked upper cervical prevertebral soft tissue swelling (arrow). (B) Sagittal computed tomography image showing distraction and anterior dislocation between the occipital condyles and the atlas (arrow). (C) Axial contrast computed tomography image showing the extent of the hematoma between the cervical spinal canal (black arrow) and the prevertebral space (white arrow). (D) Sagittal magnetic resonance T2-weighted image showing a hematoma (arrow) compressing the spinal cord. (E) The digital subtraction angiography shows the stretching of both internal carotid arteries (arrows) caused by atlanto-occipital dislocation. 

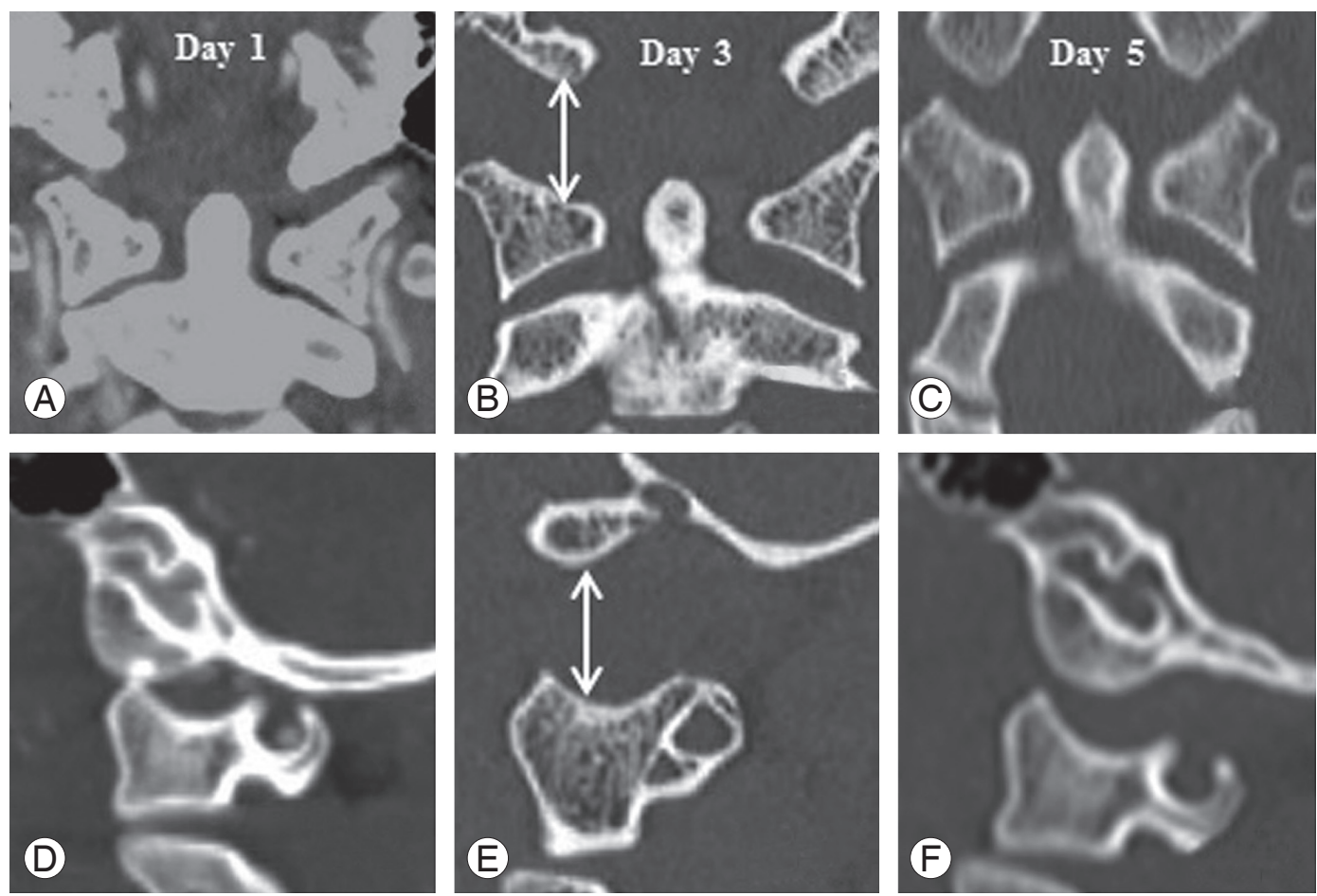

Fig. 2. Changes in the degree of dislocation shown on coronal (A-C) and sagittal (D-F) computed tomography images. Cervical stabilization was instituted with a halo vest on day 2. At day 3, however, the distraction of the craniocervical junction had further increased (arrows), accompanied by a decrease in the cerebral regional saturation of oxygen $\left(\mathrm{rSO}_{2}\right)$ value. Therefore, a corrective compressive force was attempted with the halo vest. This improved the dislocation and the patient's conscious state improved.

force to the head. This succeeded in reducing the AOD and the patient's level of consciousness improved (Fig. 2).

On the 16th day, the patient's GCS was E4V(T)M6 and the MMT score was 5 for the main muscles except the proximal upper limb muscles with a MMT score of 2. An occipito-axial posterior fixation was performed which had been delayed because an acute phase fixation surgery was considered to carry additional risks for the patient such as intraoperative hemorrhage. The patient was placed in the prone position with a halo. A connector was used to fix the halo ring to a Mayfield arm from the bed. During surgery, the atlanto-occipital gap was found to be wide. Adequate curettage and bone grafting were performed. C2 pedicle screws and an occipital plate were placed. During final fixation with a rod on each side, the Mayfield device was unlocked for manual compression of the halo, restoring an occipital-C2 congruency (Fig. 3). Following surgery, a hard collar was substituted for the halo.

The patient was able to walk without support within 2 months. Although he retained right proximal upper limb motor weakness, he eventually fully regained the ability to perform activities of daily living. Additionally, the abducens nerve palsy completely resolved.

\section{Discussion}

AOD is caused by a combination of considerable forces and patients often present with systemic traumatic damage. As a result, $A O D$ is associated with a significant mortality rate [3]. Indeed, on postmortem examination, AOD is found in $20 \%$ to $30 \%$ of patients who die of cervical spine injuries $[7,8]$. However, with the recent progress in pre-hospital care, increasing numbers of survivors are being reported $[1,2,4-6]$. Therefore, clinicians should be informed on the prompt identification and management of AOD.

CT and MRI are essential in the diagnosis of AOD. The specific landmarks and indices for AOD are clearly demonstrated on CT $[1,3,6,9]$ including the Powers ratio, the $\mathrm{X}$-line method, the basion-dens and basion-axial intervals and the condyle-C1 interval (Fig. 4) [3]. A MRI enables clinicians to evaluate a breakage of the supporting liga- 

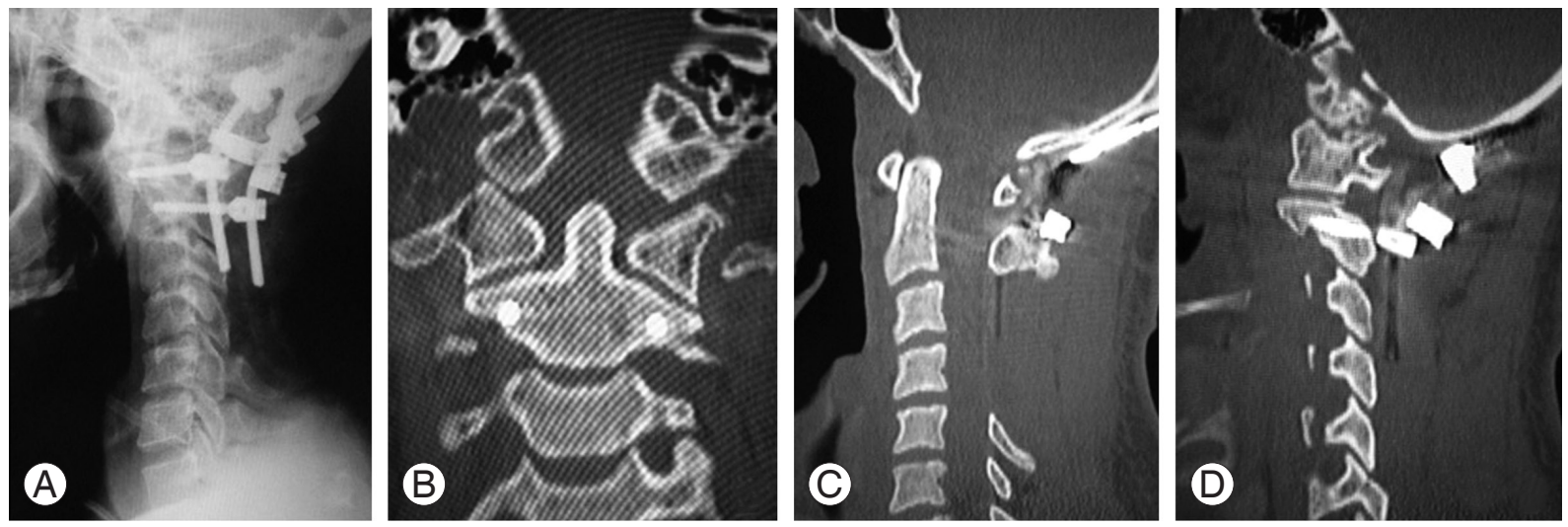

Fig. 3. Postoperative radiological findings. Lateral X-ray results (A) showing occipital-C2 posterior fixation using an occipital plate, C2 pedicle screws, rods and a crosslink. Coronal (B) and sagittal (C, D) computed tomography images showing a reduction of the atlanto-occipital dislocation.
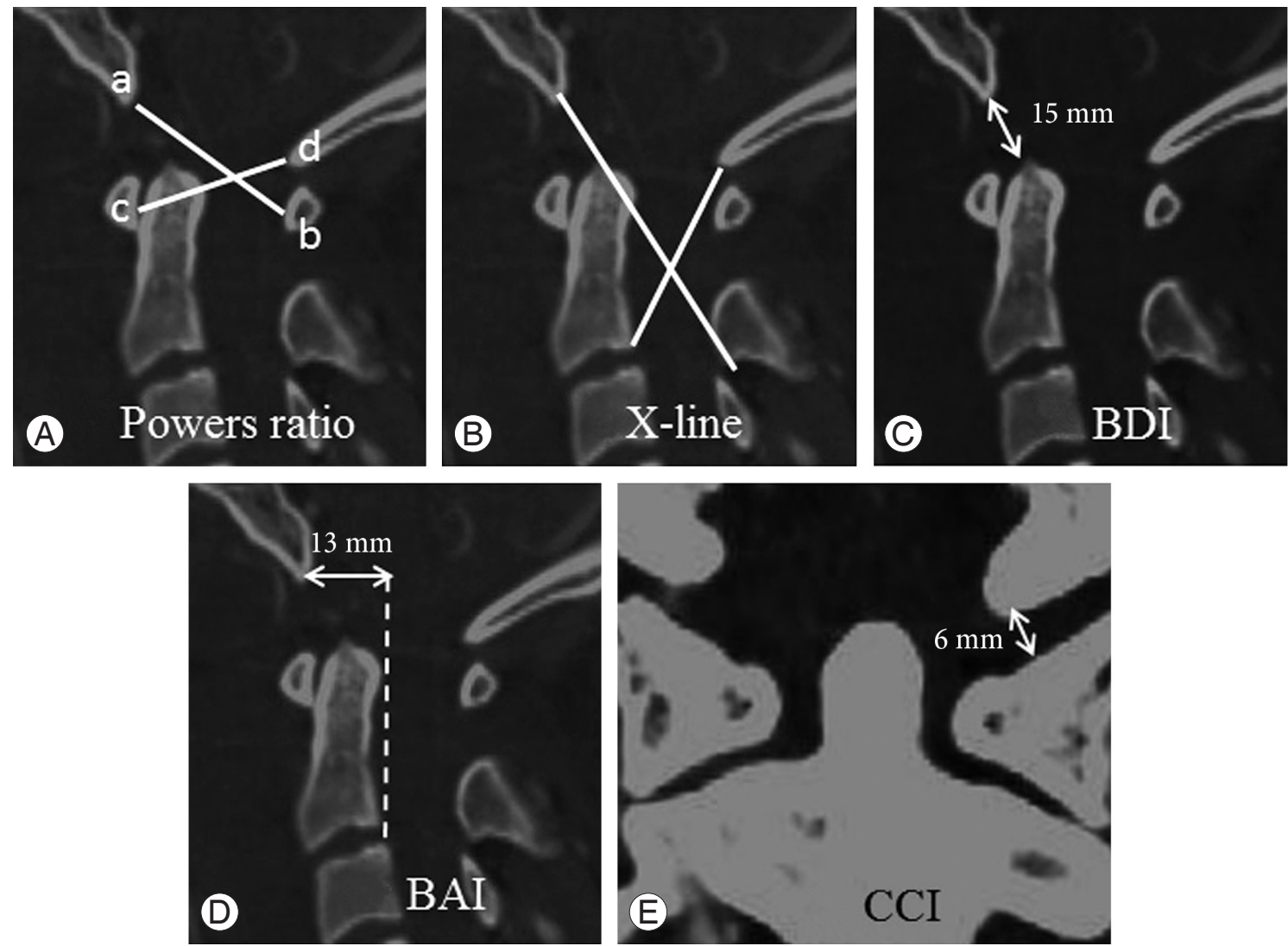

Fig. 4. Indices of atlanto-occipital dislocation in the present case. The Powers ratio is the ratio of the basion-posterior atlas arch to the opisthion-anterior arch (ab/cd); values greater than 1 are abnormal. In our case, the Powers ratio was 1.2 (A). According to the X-line method, an abnormality is present if the line from the basion to the axis spinolaminar junction does not intersect C2 and a line from the opisthion to the posteroinferior corner of the body of the axis does not intersect C1. The latter criterion was met in the present case (B). The basion-dens interval (BDI) is abnormal in the presence of a displacement between the basion and the dens of greater than $10 \mathrm{~mm}$ in adults or greater than $12 \mathrm{~mm}$ in pediatric patients. The BDI was $15 \mathrm{~mm}$ in the present case (C). An anterior displacement of at least $12 \mathrm{~mm}$ or a posterior displacement of at least $4 \mathrm{~mm}$ between the basion and the posterior $\mathrm{C} 2$ line denotes an abnormal basion-axial interval (BAI). A 13-mm anterior displacement was seen in the present case (D). A condyle-C1 interval (CCI), which is the distance between the occipital condyle and the superior articular facet of the atlas, of more than $2 \mathrm{~mm}$ in adults or more than $5 \mathrm{~mm}$ in pediatric patients is considered to be abnormal. The CCI was $6 \mathrm{~mm}$ in the present case (E). 
Table 1. Grades of atlanto-occipital dislocation

\begin{tabular}{lll} 
Grade & \multicolumn{1}{c}{ Computed tomography } & \multicolumn{1}{c}{ Magnetic resonance imaging } \\
\hline I & $\begin{array}{l}\text { Normal findings based on established methods } \\
\text { (Powers ratio, BDI, BAI, X-line, CCI) }\end{array}$ & $\begin{array}{l}\text { Moderately abnormal findings (high signal in posterior ligaments } \\
\text { or atlanto-occipital joint) }\end{array}$ \\
\hline II & $\begin{array}{l}\text { One or more abnormal findings based on established } \\
\text { methods }\end{array}$ & $\begin{array}{l}\text { Grossly abnormal findings in atlanto-occipital joint, tectorial } \\
\text { membrane, alar ligaments, or cruciate ligaments }\end{array}$ \\
\hline
\end{tabular}

Grades are according to Horn et al. [6].

$\mathrm{BDI}$, basion-dens interval; BAI, basion-axial interval; $\mathrm{CCl}$, condyle-C1 interval.

ments and connective tissues of the junction $[1,3,6,9,10]$. It can also identify a spinal cord injury. In addition, CT angiography, magnetic resonance angiography and DSA are essential for investigating damage to the cervical vessels.

Once AOD is suspected, a halo vest is more appropriate than a rigid cervical collar and axial traction because of the high risk of distraction at the craniocervical junction $[1,3,11]$. However, a halo orthosis is not always safe. Van de Pol et al. [9] reported re-dislocation in the presence of a halo vest in a patient with AOD. In this case, a halo orthosis was unable to provide enough compression force to immobilize the lesion because of the instability of a displaced atlanto-occipital junction [9]. Thus, frequent radiographs of the cervical spine and CT should be performed to check for a deterioration of AOD.

In the current case, cerebral $\mathrm{rSO}_{2}$ values correlated with the degree of distraction at the craniocervical junction. Distraction stretches the arteries, reducing blood flow and inducing drowsiness. The patient also developed abducens nerve paralysis as the distraction increased. This has been previously reported under the same conditions $[1,11]$ and can be a warning sign of insufficient cerebral perfusion in patients with AOD.

A grading system for patients with AOD that is useful in the assessment of surgery indications has been proposed by Horn et al. (Table 1) [6]. Grade I dislocations can be managed with conservative observation and an external orthosis, whereas Grade II dislocations require internal fixation $[3,6]$.

Occipito-cervical short fusion is the gold standard surgical treatment for AOD. However, the fusion should be extended to at least $\mathrm{C} 2$, because the major ligaments of the atlanto-occipital connection are also responsible for the competency between $\mathrm{C} 1$ and $\mathrm{C} 2$. The reported case illustrates that prompt identification and initial stabilization of AOD, followed by definitive surgical fixation can lead to a good long-term outcome.

\section{Conflict of Interest}

No potential conflict of interest relevant to this article was reported.

\section{References}

1. Steinmetz MP, Lechner RM, Anderson JS. Atlantooccipital dislocation in children: presentation, diagnosis, and management. Neurosurg Focus 2003;14:ecp1.

2. Bani A, Gilsbach JM. Atlantooccipital distraction: a diagnostic and therapeutic dilemma: report of two cases. Spine (Phila Pa 1976) 2003;28:E95-7.

3. Garrett M, Consiglieri G, Kakarla UK, Chang SW, Dickman CA. Occipitoatlantal dislocation. Neurosurgery 2010;66:48-55.

4. Chaput CD, Torres E, Davis M, Song J, Rahm M. Survival of atlanto-occipital dissociation correlates with atlanto-occipital distraction, injury severity score, and neurologic status. J Trauma 2011;71:393-5.

5. Ehlinger M, Charles YP, Adam P, et al. Survivor of a traumatic atlanto-occipital dislocation. Orthop Traumatol Surg Res 2011;97:335-40.

6. Horn EM, Feiz-Erfan I, Lekovic GP, Dickman CA, Sonntag VK, Theodore N. Survivors of occipitoatlantal dislocation injuries: imaging and clinical correlates. J Neurosurg Spine 2007;6:113-20.

7. Alker GJ Jr, Oh YS, Leslie EV. High cervical spine and craniocervical junction injuries in fatal traffic accidents: a radiological study. Orthop Clin North Am 1978;9:1003-10.

8. Bohlman $\mathrm{HH}$. Acute fractures and dislocations of the cervical spine: an analysis of three hundred hospitalized patients and review of the literature. J Bone Joint Surg Am 1979;61:1119-42. 
9. van de Pol GJ, Hanlo PW, Oner FC, Castelein RM. Redislocation in a halo vest of an atlanto-occipital dislocation in a child: recommendations for treatment. Spine (Phila Pa 1976) 2005;30:E424-8.

10. Sun PP, Poffenbarger GJ, Durham S, Zimmerman
RA. Spectrum of occipitoatlantoaxial injury in young children. J Neurosurg 2000;93:28-39.

11. Evarts CM. Traumatic occipito-atlantal dislocation. J Bone Joint Surg Am 1970;52:1653-60. 